\title{
Evaluation of a Short Term Education Program in Rural Southern Ethiopia
}

\author{
Mia Gonzales ${ }^{1}$, Cameron Nichols ${ }^{1}$, Evelyn Ashiofu ${ }^{1}$, Patrick Butler $^{1}$, Christine Zink $^{1} \&$ Christopher McNeil $^{1}$ \\ ${ }^{1}$ School of Medicine, University of Texas Health Science Center-San Antonio, USA \\ Correspondence: Christine Zink, 18502 Eagle Ford, San Antonio, TX 78258, USA. Tel: 210-730-2393. E-mail: \\ professorzinky@gmail.com
}

Received: December 30, 2016 Accepted: February 14, 2017 Online Published: February 28, 2017

doi:10.5539/gjhs.v9n4p212 URL: https://doi.org/10.5539/gjhs.v9n4p212

\begin{abstract}
Trachoma, a bacterial infection of the eye, remains the leading cause of preventable blindness worldwide. Education programs help curb the spread of disease and several programs are student-led. This study evaluates a similar short-term education program focused on transmission and prevention of trachoma.

A one-week education program was enacted by students from Texas focusing on prevention and transmission of trachoma. Populations included 30 members of an established women's group and 89 elementary students in Aleto Wondo, Ethiopia. A pretest was administered to evaluate baseline knowledge of this illness. After program completion, a posttest was administered to evaluate changes in knowledge and, thereby, effectiveness of the education program.

Survey results showed improvement in knowledge in both children and adults regarding the etiology of trachoma; average pretest to posttest scores improved from $48 \%$ to $74 \%$ correct in adolescents and from $57 \%$ to $90 \%$ correct in adults. Both groups showed improved knowledge regarding transmission of trachoma; average pretest to posttest scores improved from $39 \%$ to $95 \%$ correct in adolescents and from $34 \%$ to $83 \%$ correct in adults. The children showed improved knowledge regarding prevention of trachoma. However, we did not show a significant improvement in knowledge regarding prevention of trachoma in the adults.

Our work shows that this education program can be effective in increasing knowledge and prevention of trachoma, particularly among children. We hope that our education program can set an example of successful educational intervention for other student-led trips to endemic areas around the world.
\end{abstract}

Keywords: blindness, Chlamydia trachomatis, education, Ethiopia, SAFE, student-led, trachoma

\section{Introduction}

Trachoma, a bacterial infection caused by Chlamydia trachomatis, remains the leading cause of preventable blindness worldwide. The bacterium is transmitted via direct contact with eyes or nose of infected persons, through shared linens, and through flies that serve as carriers of the bacterium. Berhane et al. (2007) note that despite enormous strides in prevention and treatment of trachoma it continues to pose a significant burden in the rural developing world, particularly Africa. Smith et al. (2013) describe that there are 129.4 million people in Africa who live in areas that are confirmed to be trachoma endemic. There have been several studies that have looked into the burden of the disease in many countries and Dickman and Berhanu (2013) show that Ethiopia has been found to be the country with the heaviest burden, with many children suffering from trichiasis, a complication of the disease leading to introversion of eyelashes. This complication, without repair, leads to corneal abrasions, scarring and eventual blindness. To compound the problem, a study done by Lange, Baunach, McKenzie, (2014) found that leaders of rural communities in endemic areas, such as teachers and traditional healers, are not only unaware that their community is burdened by trachoma but also find it normal for children to have dirty faces that are likely harboring the bacterium. This lack of awareness by community leaders and large disease burden has been personally observed by faculty and students from the University of Texas Health Science Center at San Antonio who have been traveling to rural Ethiopia for the past eight years. These experiences in Aleto Wondo, Ethiopia, encouraged current students to develop a new education program designed to increase awareness of trachoma and its prevention strategies.

The World Health Organization (WHO) endorses the SAFE strategy to combat endemic trachoma. SAFE stands 
for Surgery for Trichiasis, distribution of Azithromycin, teaching Facial cleanliness, and improving Environmental sanitation. Since this program's inception in 1998, there has been a reduction in the prevalence of trachoma. However, Lavette, Lansingh, Carter, Eckert, Silva, (2013) indicate that there are still major concerns in the strategy including reemergence of the disease after surgery and distribution of antibiotics and incomplete understanding of the environmental factors that can lead to the disease. There have been studies that have evaluated the efficacy in using health education as a means of preventing disease. Edwards, Cumberland, Hailu, Todd, (2006) had success with the use of education, citing a significant (8\%) decrease in the prevalence of active trachoma and an overall increase in the awareness of trachoma after 12 months of follow up. In rural Tanzania, a school based curriculum was developed to help change behaviors that can help stop the transmission of the disease. After one year, follow up studies by Lewallen et al. (2008) showed a significant reduction in nasal discharge and dirty faces as well as some improvement in knowledge and behavior related indices in primary school children in intervention villages.

Based on resources available, the University of Texas Health Science Center students centered their education program around the (F) and (E) areas of the SAFE strategy. We emphasized teaching Facial cleanliness and showing individuals how to improve Environmental sanitation. Evaluation of education programs specifically in rural areas of Ethiopia is lacking in the literature. We hypothesized that our education program will significantly increase knowledge regarding transmission, burden and prevention of the disease and, in turn, will decrease prevalence of trachoma, preserve sight and enhance the quality of life in Aleto Wondo, Ethiopia. We hope that our education program can set an example of successful educational intervention for other student-led trips to endemic areas around the world.

\section{Methods}

The University of Texas Health Science Center in San Antonio (UTHSCSA) Trachoma Education Project was conducted in Aleto Wondo, Ethiopia. We partnered with Common River, a non-governmental organization that serves as a school for the local community. Common River teaches grades 1-4 and also runs an evening Women's Education Program. The UTHSCSA Center for Medical Humanities and Ethics supports Ethiopia Outreach, a student-run organization, in their annual trip to Aleto Wondo, Ethiopia. Ethiopia Outreach brings approximately ten medical students and two physicians (Figure 1) on this annual trip to provide clinical care to the local population and also to conduct education programs to enhance community health. The group focuses on cross-cultural experiences. Ethiopia Outreach is provided with transportation, lodging, and translators through Common River. 


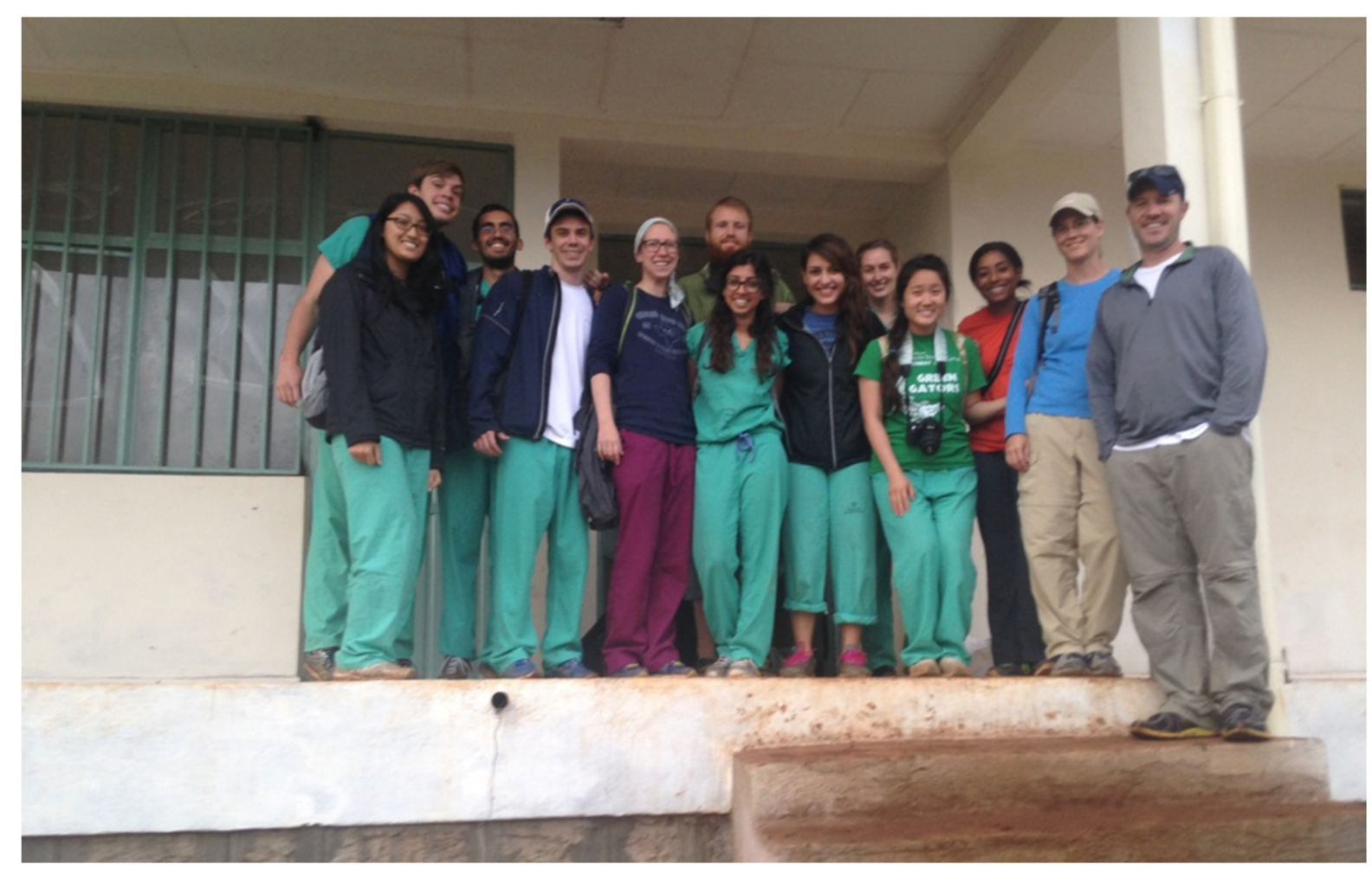

Figure 1. University of Texas Health Science at San Antonio medical students and faculty mentors

Our education project was conducted with two different groups, school-aged children and the women's group (Figure 2). The first group was formed from 89 school-aged children that attended Common River. The participants ranged from 2 nd to 4 th grade. All interaction with the participants was done via translators. The same translators were used throughout all of the education and teaching project to minimize translation variables.

Each participant was administered a pretest in order to determine baseline understanding of trachoma. The pretest was multiple-choice format spoken verbally via a translator in the participant's native language, Sidama. The pretest was administered in a large group testing setting that mimicked the testing setting for the 2nd-4th graders' examinations during school. Their teacher served as the administrator of the pretest by reading each question out loud and then allowing time for each student to write down his or her answer. All participants were given equal time to answer the questions. The surveys were then gathered and were back-translated from Sidama to English by the translators. 


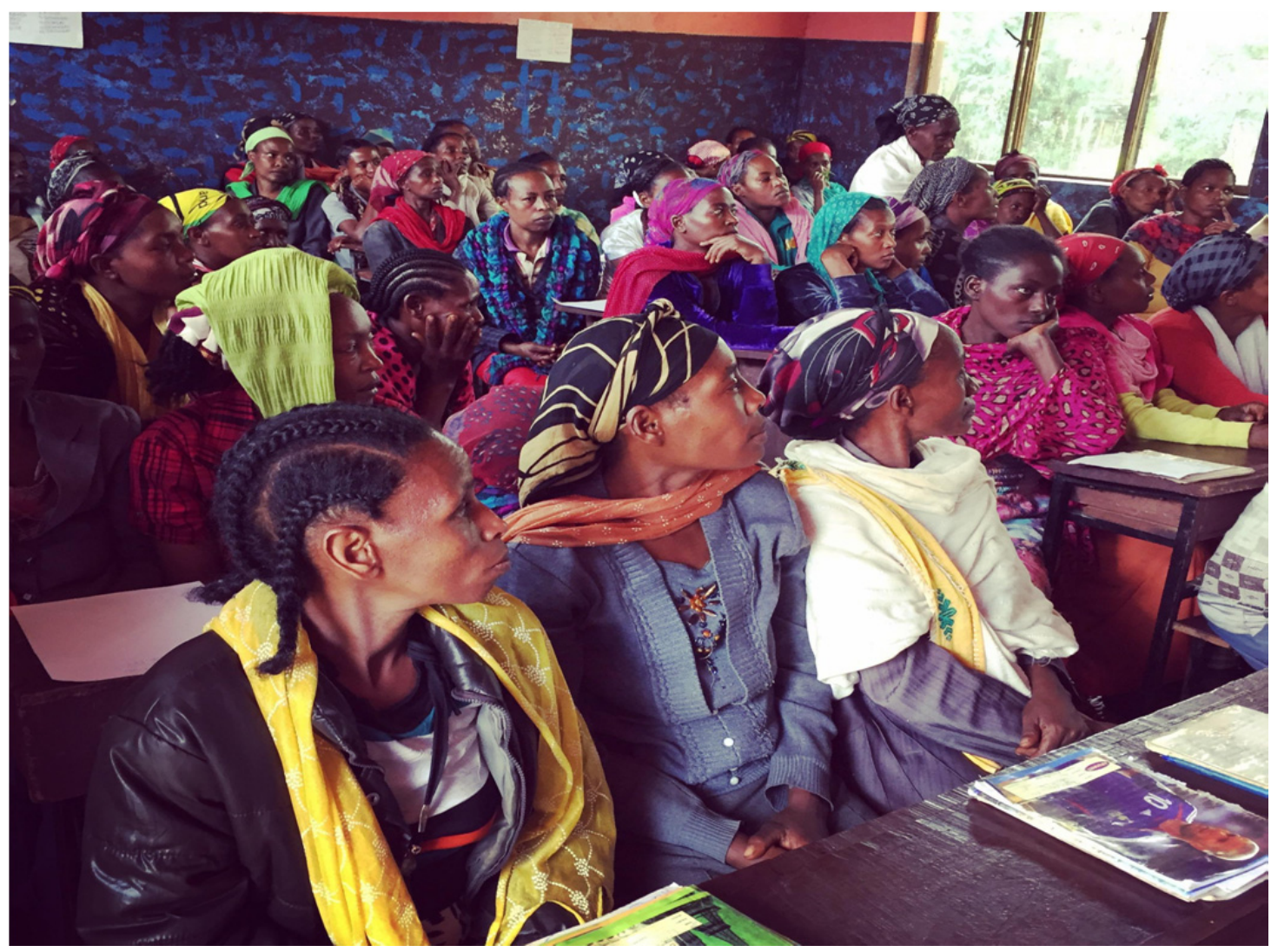

Figure 2. Adult study participants.

Following the pretest, the school-aged group received education regarding the topics covered in the pretest. The group was educated in five separate sessions over the course of five days. They were taught in an interactive manner which included songs, dancing, visual aids (picture cards), and games (Figures 3 and 4). The topics covered included facial hygiene, modes of transmission of trachoma, and prevention methods. Specifics included education on swatting flies away from the face, touching infected eyes and faces leading to subsequent transmissions, and shared cloth precautions. At the conclusion of the education sessions, the same test was administered to the group as a posttest, following the same format as the pretest.

The second group consisted of 30 women from the local community. They were primarily recruited from the Women's Education Program with an approximate age range from 40- to 60-years-old. The participants were primarily mothers and homemakers from the local community. The testing and teaching methods for this group were similar to the school-aged group, with notable differences as follows: 1) the women's group was selected based upon availability and individual willingness to participate, 2) the education seminar for the women's group was taught in a lecture-style format over a two day period resulting in approximately five hours of classroom instruction, 3) the women's group was administered the pre- and posttests in an individual format. We assumed a group answer session would create bias among the women; therefore, we chose an individual format instead. Additionally, many of the participants were illiterate and required an administrator to aid them in reading the test. The translator asked the participants questions separate from the group, then wrote down the verbal response of each participant.

Upon completion of all testing, the differences in responses of the pre- and posttests were evaluated from both groups and analyzed in order to determine the amount of knowledge gained and retained from the education program. These paired, pre- versus post-binary responses were summarized with tables of paired frequencies and percentages. The significance of the association between the pre- and post-response was assessed with McNemar's Chi-Square test for paired data. All statistical testing was two-sided with a significance level of $5 \%$. R was used throughout. 


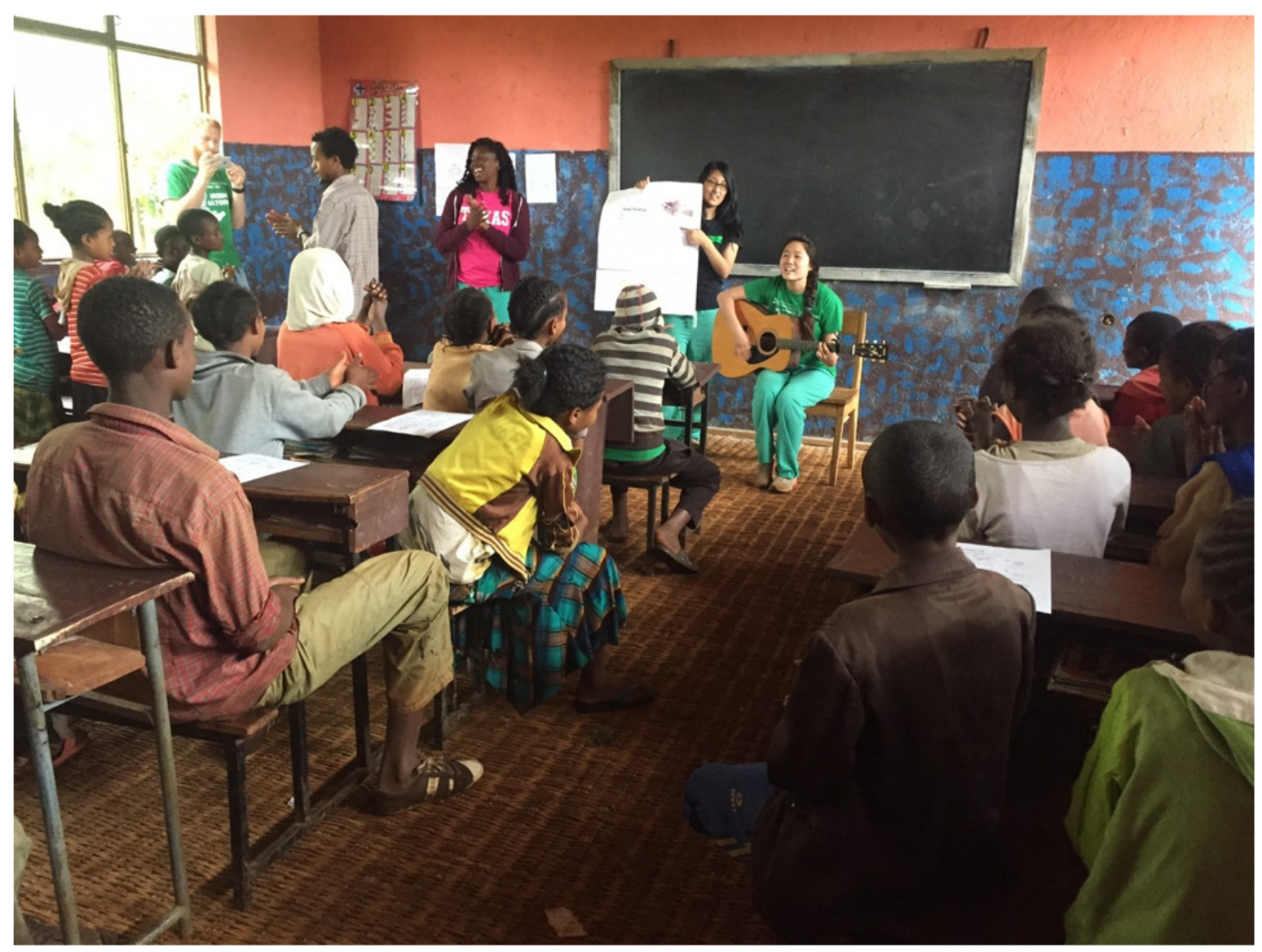

Figure 3. Medical students singing educational songs for adolescent study participants 


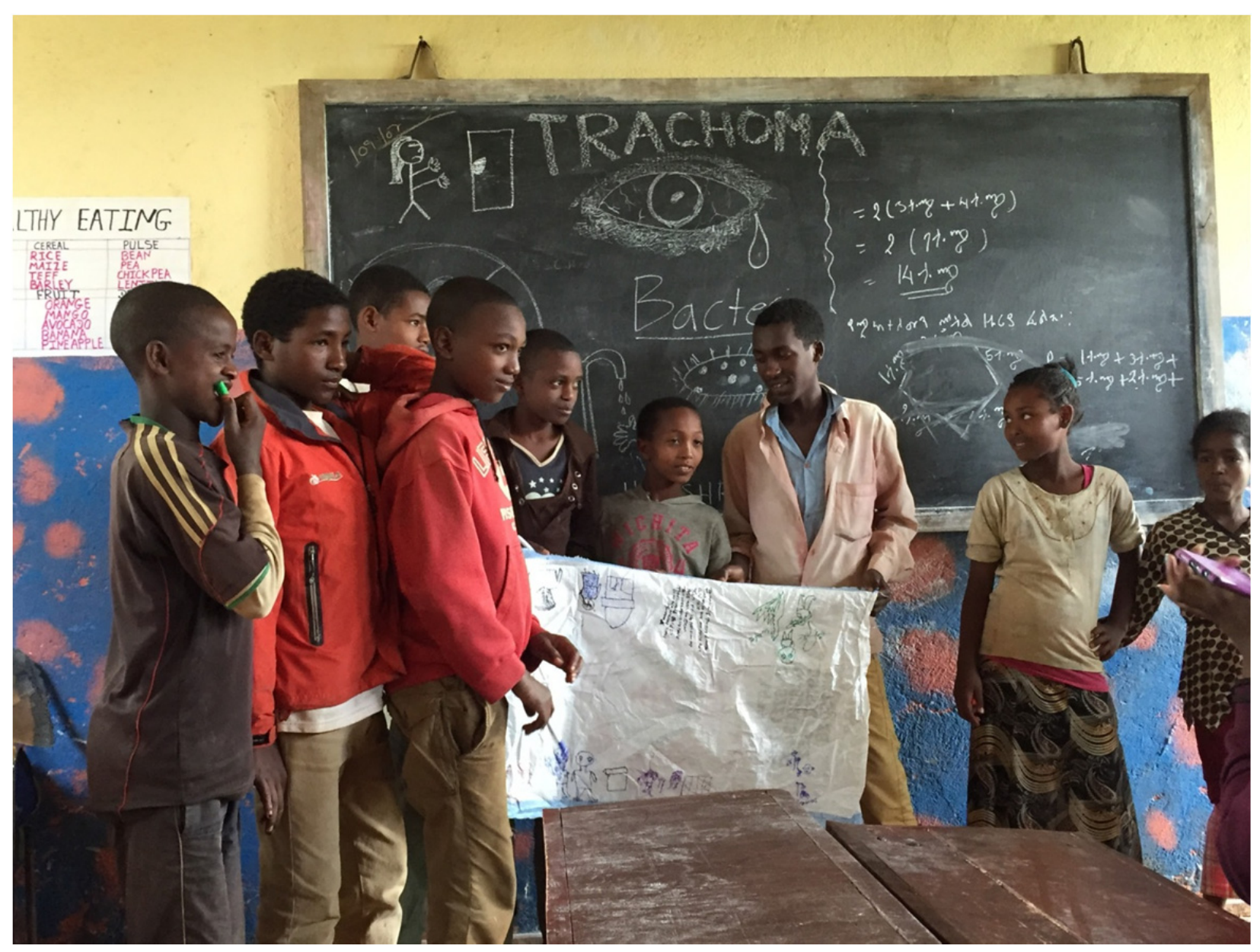

Figure 4. Adolescents describing lessons learned

\section{Results}

The results of the pre- and post-intervention surveys are depicted in Figure 5 and Tables 1 (adolescents) and 2 (adults). The first category of questions focused on the etiology of trachoma. Amongst the children who completed the pretest, only $43 \%$ of them answered correctly on 'what is trachoma', $30 \%$ answered correctly on the cause of trachoma and $70 \%$ answered correctly on whether trachoma leads to blindness. However, in the posttest, $87 \%$ $(\mathrm{p}=<0.001)$ of children answered correctly on 'what is trachoma' and $94 \%(\mathrm{p}=<0.001)$ answer correctly on whether trachoma leads to blindness. Only $42 \%(\mathrm{p}=<0.001)$ of children answered correctly on the cause of trachoma in the posttest but this was still a significant increase in knowledge regarding the etiology of trachoma.

On the other hand, in the adult population, $60 \%$ of participants answered correctly on 'what is trachoma', $43 \%$ answered correctly on the cause of trachoma and $70 \%$ answer correctly on whether trachoma leads to blindness. On the posttest, $76 \%(\mathrm{p}=.27)$ answered correctly on 'what is trachoma', 93\% $(\mathrm{p}=0.001)$ answered correctly on the cause of trachoma and $100 \%$ were correct in knowing that trachoma leads to blindness. The p-value was not obtainable due to $100 \%$ correct responses on the adult posttest.

Modes of transmission of trachoma were also a focus of the education project and were tested with the questions, 'Can trachoma be spread from person to person?', 'Can trachoma be spread by towels or clothes?' and 'Can flies on your face lead to trachoma?' Amongst the children, $48 \%$ answered correctly regarding person to person transmission, $16 \%$ answered correctly for clothing to person transmission and 53\% answered correctly regarding transmission from flies on one's face. On the posttest, $98 \%(\mathrm{p}=<0.001)$ answered correctly for person to person transmission, $90 \%(\mathrm{p}=<0.001)$ answered correctly for clothing to person transmission, and $99 \%(\mathrm{p}=<0.001)$ answered correctly regarding transmission from flies on one's face.

Amongst the adults, $30 \%$ answered correctly regarding person to person transmission, $7 \%$ answered correctly for clothing to person transmission and $67 \%$ answered correctly regarding transmission from flies on one's face. On the posttest, $77 \%(\mathrm{p}=0.001)$ answered correctly for person to person transmission, $73 \%(\mathrm{p}=<0.001)$ answered 
correctly for clothing to person transmission, and $100 \%$ answered correctly regarding transmission from flies on one's face. The p-value was not obtainable due to $100 \%$ correct responses on the adult posttest.

We evaluated prevention of trachoma and hygiene practices with the questions 'Is it normal for children to have dirty faces?', 'Do you know how to wash your face and hands?', 'Do you wash your face every day?', ' Do you talk to your family about hygiene?', 'Does good hygiene help prevent trachoma?', 'Does washing your face help prevent trachoma?' and 'Should you touch someone's face or eye if it is swollen and red?' Amongst the children 67\% answered correctly regarding whether is it normal for children to have dirty faces, $24 \%$ answered correctly regarding knowing how to wash their face and hands, $90 \%$ indicated they wash their face every day, $87 \%$ indicated they speak with their family about hygiene, $67 \%$ answered correctly regarding the prevention of trachoma with good hygiene, $91 \%$ answered correctly regarding whether washing one's face helps prevent trachoma and 55\% answered correctly whether to touch a red face or eye. On the post-test, $84 \%(\mathrm{p}=0.01)$ answered correctly regarding whether is it normal for children to have dirty faces, $90 \%(\mathrm{p}=<0.001)$ answered correctly regarding knowing how to wash their face and hands, $93 \%(\mathrm{p}=0.55)$ indicated they wash their face every day, $94 \%(\mathrm{p}=0.15)$ indicated they speak with their family about hygiene, $96 \%(\mathrm{p}=<0.001)$ answered correctly regarding the prevention of trachoma with good hygiene, $98 \%(\mathrm{p}=0.11)$ answered correctly regarding whether washing one's face helps prevent trachoma and $89 \%(\mathrm{p}=<0.001)$ answered correctly whether to touch a red face or eye.

Amongst adults, 53\% answered correctly regarding whether is it normal for children to have dirty faces, $93 \%$ answered correctly regarding knowing how to wash their face and hands, $100 \%$ indicated they wash their face every day, 93\% indicated they speak with their family about hygiene, $97 \%$ answered correctly regarding the prevention of trachoma with good hygiene, $93 \%$ answered correctly regarding whether washing one's face helps prevent trachoma and $97 \%$ answered correctly whether to touch a red face or eye. On the post-test, $77 \%(\mathrm{p}=0.1)$ answered correctly regarding whether is it normal for children to have dirty faces, $93 \%(p=1)$ answered correctly regarding knowing how to wash their face and hands, $93 \%(\mathrm{p}=1)$ indicated they speak with their family about hygiene, $100 \%$ answered correctly regarding the prevention of trachoma with good hygiene, $100 \%$ answered correctly regarding whether washing one's face helps prevent trachoma and $97 \%(p=1)$ answered correctly whether to touch a red face or eye.

Some of the p-values were not obtainable in this study because of the statistical analysis method that was used. Numerical responses were used and this created empty cells in the algorithm, when $100 \%$ for correct responses was obtained. This created an error in the p-value calculation for those responses and is marked in the table as N/A.

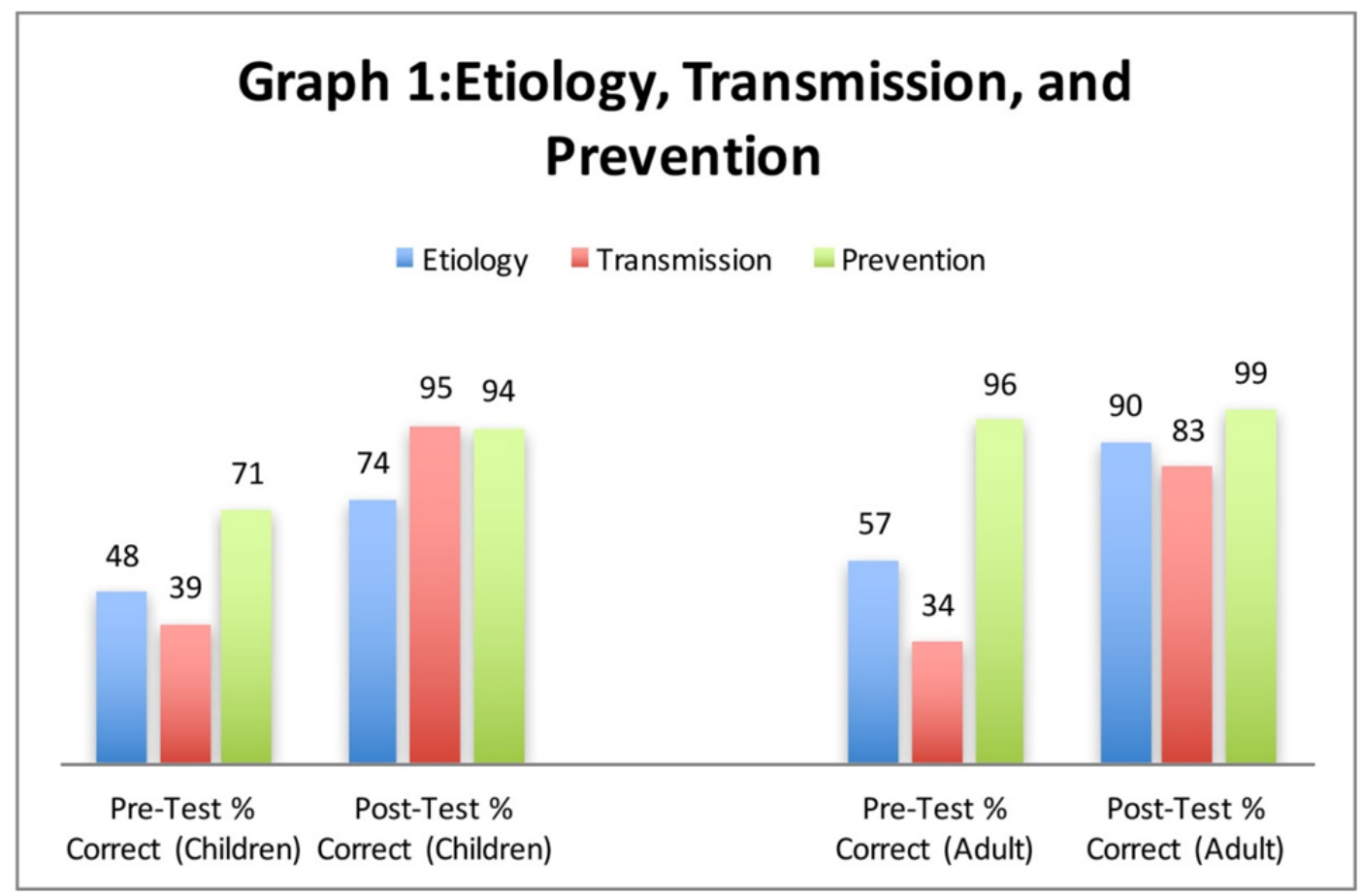

Figure 5. Pre- and posttest average test scores in both adolescence and adults in areas of etiology, transmission and prevention of trachoma 
Table 1. Adolescent Pre- versus Posttest

\begin{tabular}{lccc}
\hline Question & $\begin{array}{l}\text { Pre-Test }(\mathbf{n}=\mathbf{8 9}) \\
\text { \% Correct }\end{array}$ & $\begin{array}{l}\text { Post-Test }(\mathbf{n}=\mathbf{8 9}) \\
\text { \% Correct }\end{array}$ & p-value \\
\hline Do you know what trachoma is? & 43.33 & 87.78 & $<0.001^{1 *}$ \\
Do you know what causes trachoma? & 30.33 & 42.34 & $<0.001^{1 *}$ \\
Can trachoma lead to blindness? & 70.79 & 94.38 & $<0.001^{1 *}$ \\
Can trachoma be spread person to person? & 47.73 & 97.73 & $<0.001^{1 *}$ \\
Can trachoma be spread by towels or clothes? & 15.73 & 89.89 & $<0.001^{1 *}$ \\
Can flies on face lead to trachoma? & 53.41 & 98.86 & $<0.001^{1 *}$ \\
Is it normal for children to have dirty faces? & 67.05 & 90.91 & $0.01^{1}$ \\
Do you know how to wash your face and hands? & 23.86 & 93.18 & $<0.001^{1 *}$ \\
Do you wash your face everyday? & 89.77 & 94.38 & $0.55^{1}$ \\
Do you talk to your family about hygiene? & 86.52 & 96.67 & $0.15^{1}$ \\
Does good hygiene help prevent trachoma? & 66.67 & 97.75 & $<0.001^{1 *}$ \\
Does washing your face help prevent trachoma? & 91.01 & 88.76 & $0.11^{1}$ \\
Should you touch someone's face/eyes if it is swollen and red? & 55.06 & $<0.001^{1 *}$ \\
\hline
\end{tabular}

1. McNemar's Chi-squared Test;

${ }^{*}$ p-value $<0.05$.

Table 2. Adult Pre- versus Posttest

\begin{tabular}{|c|c|c|c|}
\hline Question & $\begin{array}{l}\text { Pre-Test }(n=30) \\
\% \text { Correct }\end{array}$ & $\begin{array}{l}\text { Post-Test }(n=30) \\
\% \text { Correct }\end{array}$ & p-value \\
\hline Do you know what trachoma is? & 60 & 76.67 & $0.27^{1}$ \\
\hline Do you know what causes trachoma? & 43.33 & 93.33 & $0.001^{1 *}$ \\
\hline Can trachoma lead to blindness? & 70 & 100 & $\mathrm{~N} / \mathrm{A}^{1}$ \\
\hline Can trachoma be spread person to person? & 30 & 76.67 & $0.001^{1 *}$ \\
\hline Can trachoma be spread by towels or clothes? & 6.67 & 73.33 & $<0.001^{1 *}$ \\
\hline Can flies on face lead to trachoma? & 66.67 & 100 & $\mathrm{~N} / \mathrm{A}^{1}$ \\
\hline Is it normal for children to have dirty faces? & 53.33 & 76.67 & $0.1^{1}$ \\
\hline Do you know how to wash your face and hands? & 93.33 & 93.33 & $1^{1}$ \\
\hline Do you wash your face everyday? & 100 & 96.67 & $\mathrm{~N} / \mathrm{A}^{1}$ \\
\hline Do you talk to your family about hygiene? & 93.33 & 93.33 & $1^{1}$ \\
\hline Does good hygiene help prevent trachoma? & 96.67 & 100 & $\mathrm{~N} / \mathrm{A}^{1}$ \\
\hline Does washing your face help prevent trachoma? & 93.33 & 100 & $\mathrm{~N} / \mathrm{A}^{1}$ \\
\hline Should you touch someone's face/eyes if it is swollen and red? & 96.67 & 96.67 & $1^{1}$ \\
\hline
\end{tabular}

1. McNemar's Chi-squared Test.

$*$ p-value $<0.05$.

\section{Discussion}

From our results we found that, not unlike other studies performed, our trachoma education project had a significant short-term impact on the etiology, transmission and prevention of trachoma in this rural Ethiopian town. The most significant increases in knowledge were identified in the areas related to the etiology and transmission of trachoma, particularly in the adolescent participants. There was less of a change in knowledge regarding prevention of trachoma, as many of the adults were already aware that good hygiene practices would prevent 
trachoma. However, in the adolescent population there was still a significant increase in understanding that good hygiene can help prevent trachoma. Dickman and Berhanu (2013) showed a 60\% increase in usage of azithromycin in a rural community who utilized education programs targeting adolescents. Therefore, we think it encouraging that the adolescents in Aleto Wondo showed improved knowledge that can be disseminated to the adult population. Providing education to children has the biggest impact in changing a community and our study shows that the adolescent participants had a significant improvement in knowledge regarding, not only the etiology and transmission of trachoma, but also prevention of the disease.

One might consider that since many adult study participants were already aware that good hygiene helps prevent trachoma that our education program might not change prevalence of the disease. However, we feel that the improved knowledge regarding etiology and transmission of trachoma will only enhance prevention strategies that have already been instituted. Edwards et al. (2006) studied awareness of trachoma after broadcast radio educational programs were instituted and then calculated the change in prevalence of the disease. They showed a significant drop in disease prevalence but only a minor improvement in behavioral changes after educational programs were instituted. They concluded that the decrease in prevalence must have been attributed to more than education alone. We also feel that our education programs are only an important aspect of change and decreased prevalence and should not be used in isolation.

\subsection{Limitations:}

Our trachoma education outreach targeted both adults and adolescents but ultimately focused on adolescents. Because there was such a large population of children who already attended school at Common River, the adolescent population were more reliable to return and tripled the size of the adult population. The smaller sample size of adults could have accounted for some of the non-significant changes observed from pre- to posttest questions. Fortunately, Dickman and Berhanu's (2013) work suggests that educating children can significantly affect the population that they live in, thereby reinforcing the inclination to accept such a large population of adolescents in this survey.

The education that was provided by our team followed the daily school lessons which greatly improved participation by the younger population. However, the adults were taught during the time when they would normally be receiving life skills classes in the evening. Due to issues such as inclement weather and employment commitments, study participation amongst the adult population could not be maintained as tightly as the adolescent population. Another issue to be considered is that the adolescents in this study are accustomed to spending time in a classroom setting. The adults in this study were not raised in a traditional education setting so these differences may have affected our pre- and posttest results. Finally, all education and evaluations were done via translator in the classroom. Since the translators had to translate from English to Sidama and then back to English to answer the pre- and posttest questions, certain questions or answers could have been misinterpreted.

\section{Conclusion}

We have been building relationships in Aleto Wondo, Ethiopia for over eight years and we hope that those bonds will help us serve the community better. We have fostered an environment of trust and open communication for cultural exchange and this has afforded the opportunity to implement worthwhile educational programs. We have shown that a short-term education program targeting etiology, transmission and prevention of trachoma improves knowledge in adolescents and adults in a rural Ethiopian community. These findings and the overall experiences during the program not only benefit the local community but also form lasting impressions on young American medical students conducting life altering work. More work needs to be done to truly determine the impact of our education program in Aleto Wondo, Ethiopia. The long-term effectiveness of our project will be better understood after the results of follow-up surveys conducted at 1-year have been completed. We also hope to use this model to develop other education programs for preventable illnesses.

\section{Acknowledgements}

Thank you to the Center for Humanities and Ethics for support of Ethiopia Outreach.

\section{Competing Interests Statement}

The authors declare that there is no conflict of interests regarding the publication of this paper.

\section{References}

Berhane, Y., Worku, A., Bejiga, A., Adamu, L. A., Wondu, A., Bedri, A., West, S., et al. (2007). Prevalence of Trachoma in Ethiopia. Ethiopian Journal of Health Promotion, 21(3), p211-215.

Dickman, L., \& Berhanu, M. (2013). Children as Agents of Change in Trachoma Control. Community Eye Health 
Journal, 26(81), 18.

Edwards, T., Cumberland, P., Hailu, G., \& Todd, J. (2006) Impact of Health Education on Active Trachoma in Hyperendemic Rural Communities in Ethiopia. Ophthamology, 113(4), p548-555. https://doi.org/10.1016/j.ophtha.2006.01.008

Lange, F., Baunach, E., McKenzie, R. T. (2014). Trachoma Elimination in Remote Indigenous Northern Territory Communities: a Baseline Health Promotion Study. Australian Journal of Primary Health, 20(1), p34-40. https://doi.org/10.1071/PY12044

Lavette, D., Lansingh, V., Carter, M., Eckert, K., \& Silva, J. (2013) Will the SAFE Strategy Be Sufficient to Eliminate Trachoma by 2020? Puzzlements and Possible Solutions. Scientific World Journal, p1-18. https://doi.org/10.1155/2013/648106

Lewallen, S., Massae, P., Tharaney, M., Somba, M., Geneau, R., MacArthur, C., \& Courtright, P. (2008) Evaluating a School-based Trachoma Curriculum in Tanzania. Health Education Research, 23(6), p1068-1073. https://doi.org/10.1093/her/cym097

Smith, J., Flueckiger, R., Hooper, P., Polack, S., Cromwell, E., Palmer, S., Brooker, S, et al. (2013) The Geographical Distribution and Burden of Trachoma in Africa. PLOS Neglected Tropical Disease, 7(8), p1-13. https://doi.org/10.1371/journal.pntd.0002359

\section{Copyrights}

Copyright for this article is retained by the author(s), with first publication rights granted to the journal.

This is an open-access article distributed under the terms and conditions of the Creative Commons Attribution license (http://creativecommons.org/licenses/by/4.0/). 